\title{
A ASSOCIAÇÃO ENTRE ENDOMETRIOSE E INFERTILIDADE FEMININA: UMA REVISÃO DE LITERATURA
}

\author{
THE ASSOCIATION BETWEEN ENDOMETRIOSIS AND FEMALE \\ INFERTILITY: A LITERATURE REVIEW
}

\author{
Duarte, A.N ${ }^{1}$, Righi, M.G ${ }^{2}$ \\ Afiliações: 1- Curso de Medicina da Universidade Estadual do Oeste do Paraná - UNIOESTE, Centro de Ciências da Saúde (CCS), campus \\ de Francisco Beltrão. Acadêmica do $5^{\circ}$ ano do curso de Medicina. \\ 2- Curso de Medicina da Universidade Estadual do Oeste do Paraná - UNIOESTE, Centro de Ciências da Saúde (CCS), campus de \\ Francisco Beltrão. Docente e preceptor da disciplina de Ginecologia e Obstetrícia. \\ Endereço do autor de correspondência: Rua Maranhão 978 - Vila Nova, Francisco Beltrão, Paraná, Brasil. CEP: 85601-310. \\ amandanduarte@hotmail.com
}

\section{Resumo}

Endometriose é uma doença crônica que afeta cerca de $20 \%$ das mulheres em idade fértil causada por implantes de tecido endometrial localizados fora da cavidade uterina, principalmente em locais como ovários e cavidade peritoneal. A origem dessa condição ainda permanece desconhecida, existindo na literatura diversas teorias que objetivam explicar a fisiopatologia envolvida, como por exemplo a Teoria de Sampson, conhecida como teoria da menstruação retrógrada. A doença apresenta dois desfechos principais, sendo eles: dor pélvica e infertilidade. A dificuldade de concepção espontânea está relacionada à resposta inflamatória local gerada pelas células endometriais ectópicas, o que leva a aumento nos níveis de citocinas, interleucinas, fatores de crescimento, fatores angiogênicos e células de defesa. Esse ambiente pode alterar mecanismos fisiológicos envolvidos na ovulação, fecundação e implantação, além de prejudicar a qualidade dos espermatozoides e promover alterações anatômicas do trato genital feminino. $O$ presente estudo teve como objetivo reunir os dados existentes na literatura contemporânea para compreender os mecanismos envolvidos na infertilidade gerada pela endometriose. Assim, concluiu-se que a inflamação é o evento base para todos os processos envolvidos que podem influenciar na fertilidade e que os estudos acerca do tema elevam a expectativa para o desenvolvimento de novas abordagens dessas pacientes.

Palavras-chave: endometriose; infertilidade; inflamação; dismenorreia; gravidez.

\begin{abstract}
Endometriosis is a chronic disease that affects about $20 \%$ of women in fertile age caused by implants of endometrial tissue located outside the uterine cavity, especially in places such as the ovaries and peritoneal cavity. The origin of this condition remains unknown and there are several theories in literature that aim to explain the pathophysiology involved, such as Sampson's Theory, known as retrograde menstruation theory. The disease has two main outcomes: pelvic pain and infertility. The difficulty in spontaneous conception is related to the local inflammatory response generated by ectopic endometrial cells, which leads to increased levels of cytokines, interleukins, growth factors, angiogenic factors and defense cells. This environment can alter physiological mechanisms involved in ovulation, fertilization and implantation, in addition to impairing the quality of sperm and promoting anatomical changes in the female genital tract. The present study aimed to gather the existing data in the contemporary literature to understand the mechanisms involved in the infertility caused by endometriosis. Thus, it was concluded that inflammation is the main event for all the processes involved that are able to influence fertility and that studies on the subject raise expectations for the development of new approaches for these patients.
\end{abstract}

Keywords: endometriosis; infertility; inflammation; dysmenorrhea; pregnancy. 


\section{Introdução}

Define-se endometriose como uma doença ginecológica crônica de caráter inflamatório causada por implantes de tecido endometrial dos tipos histológicos glandular e estromal localizados fora da cavidade uterina ${ }^{1}$. Apesar de bem caracterizada a relação de interdependência entre o nível de estrogênio circulante e o crescimento dos focos de endométrio ectópico, a patogênese envolvida na doença ainda permanece controversa, sendo relatadas na literatura diversas teorias que objetivam esclarecer a origem dos implantes e os possíveis fatores que atuam como influenciadores do processo. De maneira geral, elas são categorizadas em teorias que propõem a origem dos focos no endométrio uterino e teorias que colocam tecidos extrauterinos como originários destes ${ }^{2}$. Em relação ao primeiro grupo, destaca-se a Teoria de Sampson, também conhecida como teoria da menstruação retrógrada, pautada na ocorrência de episódios de refluxo menstrual por meio das tubas uterinas durante as contrações do miométrio com consequente disseminação intraperitoneal de células endometriais funcionais que, em reposta a estímulos hormonais, geram uma complexa reação inflamatória crônica que é capaz de alterar mecanismos fisiológicos ligados à capacidade reprodutiva e gerar infertilidade como uma das manifestações comuns da doença ${ }^{3}$. Entretanto, cerca de $75 \%$ a $90 \%$ das mulheres apresentam o fenômeno da menstruação retrógrada, porém apenas uma parcela desenvolve endometriose, deixando incerta a relação entre os episódios de refluxo e possíveis fatores genéticos, ambientais, bioquímicos e imunológicos que possam influenciar no advento da doença ${ }^{1}$. Além desta, teorias contemporâneas descrevem a origem dos implantes endometriais na implantação e diferenciação de células tronco embrionárias migrantes tanto da camada basal do tecido endometrial quanto da medula óssea para a cavidade peritoneal gerando os focos ectópicos ${ }^{4}$.

A real prevalência da endometriose na população feminina não é exata, porém dados da literatura mais recente apontam para uma taxa de cerca de $5 \%$ a $10 \%$ das mulheres em idade reprodutiva ${ }^{5}$. Ademais, aproximadamente $35 \%$ a $50 \%$ das mulheres diagnosticadas com a doença apresentam também quadro de infertilidade ${ }^{6}$.

Fatores de risco para o desenvolvimento da endometriose incluem o período da menacme, índice de massa corporal reduzido, mulheres 
pertencentes a classes socais mais elevadas, porém com outros fatores influentes nesse aspecto como etnia e acesso aos serviços de saúde $^{1}$, história reprodutiva relacionada à menarca precoce, ciclos menstruais curtos e intensos, além de nuliparidade ${ }^{7}$ Outro importante fator descrito é o reconhecimento de história familiar positiva para endometriose por meio de anamnese detalhada, destacando possível influência genética no desenvolvimento do quadro. Por fim, estudos recentes apontam um maior risco de desenvolver a doença em mulheres portadoras de desordens imunológicas como, por exemplo, artrite reumatoide, lúpus eritematoso sistêmico, desordens tireoidianas e esclerose sistêmica?

O quadro clínico manifesta-se mais comumente através de dor pélvica crônica, na forma de dismenorreia e/ou dispareunia de profundidade ou ainda através de infertilidade ou dificuldade de concepção ${ }^{1,8}$, a qual assume caráter multifatorial, com diferentes mecanismos que podem interferir na reprodução fisiológica, desde distorções anatômicas devido a adesões e fibrose até anormalidades do sistema endócrino e distúrbios imunológicos ${ }^{1,6}$.

Nesse sentido, a literatura aponta a endometriose como doença de gravidade progressiva, sendo que parece existir associação entre a extensão desta e o grau de redução da fertilidade espontânea, porém com força de associação variável entre os estudos ${ }^{1}$.

O diagnóstico da endometriose é realizado através do quadro clínico, exame ginecológico e exames complementares como ultrassonografia transvaginal ou ressonância magnética pélvica, sendo a laparoscopia considerada o padrão-ouro, uma vez que apenas por meio da biópsia dos focos suspeitos e posterior análise anatomopatológica é possível confirmar a hipótese diagnóstica ${ }^{9}$. A partir da realização do diagnóstico, pode-se classificar a endometriose com base no grau de acometimento da doença, objetivando correlacionar possíveis desfechos favoráveis ou não quanto às opções de tratamento disponíveis, além de permitir o seguimento das pacientes, universalizar a linguagem utilizada por profissionais no manejo do quadro, padronizar parâmetros de comparação e facilitar aplicação de pesquisas clínicas da área ${ }^{5}$.

$$
\text { A classificação mais utilizada }
$$
atualmente é da American Fertility Society (atual American Society for Reproductive Medicine ASRM), proposta no ano de 1979 e com última atualização em 1996, que classifica a 
endometriose em mínima, leve, moderada e severa, por meio de escores obtidos através da observação sistemática da pelve por laparotomia ou laparoscopia ${ }^{10}$.

O tratamento deve ser individualizado, considerando primariamente o objetivo terapêutico sendo o alívio da sintomatologia relacionada à dor pélvica crônica ou ao quadro de infertilidade causado pela doença ${ }^{9}$. Quanto à infertilidade, o tratamento é baseado em três modalidades: medicamentosa, cirúrgica e reprodução assistida, sempre levando em conta aspectos fundamentais para a decisão terapêutica como escolha da paciente, idade, status de reserva ovariana, extensão da doença, presença de dor pélvica, endometriomas, histórico de intervenções cirúrgicas prévias, presença ou ausência de anormalidades tubárias e qualidade seminal do parceiro ${ }^{11}$.

Em face do exposto, o presente estudo tem como objetivo principal identificar fatores de associação entre a endometriose e o desenvolvimento de infertilidade nas pacientes acometidas, buscando elucidar a patogênese envolvida no processo e identificar possíveis aspectos modificadores deste.

\section{Métodos}

Trata-se de uma revisão de literatura classificada como narrativa, executada por meio da análise de estudos contemporâneos acerca dos temas propostos buscando realizar um levantamento bibliográfico das principais informações disponíveis em livros, publicações de estudos em revistas científicas, artigos, trabalhos de conclusão de curso, dissertações, teses e bancos de dados como Scientific Electronic Library Online (Scielo), Literatura Latino-Americana e do Caribe em Ciências da Saúde (LILACS), PubMed (MEDLINE), UpToDate e Google Acadêmico. Foram selecionados 30 artigos utilizando os seguintes descritores: endometriose, infertilidade, inflamação, dismenorreia e gestação.

Os artigos selecionados compreendem somente descrições nas línguas portuguesa e inglesa. O período de publicação dos estudos analisados é situado entre os anos de 1990 até 2020, destacando-se aqueles publicados a partir do ano de 2010. Dessa forma, os dados obtidos por meio da pesquisa foram analisados e agrupados de forma a sintetizar as principais informações atuais disponíveis acerca do tema. 


\section{Desenvolvimento}

Infertilidade é definida como a incapacidade de um casal conseguir uma gravidez espontânea após doze meses de relações sexuais regulares e frequentes - cerca de duas a três relações por semana, excluindo-se o uso de qualquer método de anticoncepção, sendo a ausência de ovulação a causa mais comum de infertilidade relacionada à mulher ${ }^{12}$.

A associação entre endometriose e infertilidade é bem estabelecida por meio de dados provenientes da literatura, porém a exata relação de causa e efeito ainda é controversa ${ }^{13}$. A taxa de fecundidade de casais em idade reprodutiva é estimada em cerca de $15 \%$ a $20 \%$, enquanto a taxa em mulheres com endometriose não tratada é estimada em $2 \%$ a $10 \%{ }^{13}$. Estudos contemporâneos sugerem que em um grupo de mulheres com endometriose classificada como mínima e leve, de acordo com a classificação da ASRM, cerca de 50\% conseguirá engravidar sem nenhum tipo de tratamento, já em mulheres com grau moderado, apenas $25 \%$ irá conceber espontaneamente e apenas algumas conceberão em casos de doença severa ${ }^{13}$. Além disso, em relação à localização e forma de apresentação da doença, os implantes peritoneais são mais relacionados ao quadro de infertilidade quando comparados aos casos de endometriose profunda e aos endometriomas ovarianos ${ }^{14}$. Nessa perspectiva, aceita-se que a infertilidade ligada à doença assuma caráter multifatorial, com diferentes mecanismos que podem interferir no processo de reprodução normal, desde grosseiras distorções anatômicas que alteram a forma fisiológica dos órgãos pélvicos femininos devido a adesões e fibroses até anormalidades relacionadas ao sistema de regulação endócrina e distúrbios imunológicos ${ }^{14,15}$.

$$
\text { Inflamação peritoneal crônica é um }
$$
achado característico em pacientes com endometriose $^{14}$, mesmo em sua forma leve, uma vez que células endometriais quando situadas fora da cavidade uterina são identificadas como antígenos e submetidas à resposta imunológica local ${ }^{16}$. Assim, estudos atuais propõem que os implantes de endométrio localizados na cavidade peritoneal são capazes de induzir uma reação inflamatória associada à expressão aberrante de citocinas como fator de necrose tumoral alfa (TNF-alfa), interleucina 1 beta (IL-1- $\beta$ ), interleucina 6 (IL-6), interleucina 8 (IL-8), interleucina 10 (IL-10), interleucina 17 (IL-17), interleucina 33 (IL-33), proteína induzida 10 
(IP-10), proteína quimiotática de macrófagos 1 $(\mathrm{MCP}-1)^{6}$, liberação aumentada de espécies reativas de oxigênio, fatores de crescimento, fatores angiogênicos, além da proliferação, ativação e disfunção fagocitária de macrófagos, aumento da concentração e alteração funcional de células natural killers (NK) e linfócitos $\mathrm{T}^{15}$. A inflamação peritoneal gerada por meio da resposta imune afeta diversas etapas do processo de ovulação, fecundação e implantação ${ }^{11,13,15}$. No que diz respeito à ovulação, espécies reativas de oxigênio podem alterar o processo de foliculogênese e promover danos celulares reduzindo a qualidade dos oócitos liberados a cada ciclo ${ }^{17}$. Ademais, o transporte de gametas por meio das tubas uterinas também é prejudicado por conta do contato direto dessas com o fluido peritoneal inflamatório, condição que leva à disfunção de células ciliares, formação de aderências fibróticas e surgimento de contrações miometriais descoordenadas causadas pela inflamação local ${ }^{13,14}$.

Esse microambiente inflamatório possui ainda a capacidade de interferir na fertilidade por meio de mecanismos específicos como disfunção espermática e efeito deletérios na implantação. Interleucinas 1 e 6 afetam diretamente a motilidade dos espermatozoides, enquanto TNF-alfa pode causar dano ao seu DNA, e ambos, juntamente ao estresse oxidativo gerado localmente, podem interferir no processo de capacitação e reduzir o poder de ligação destes à zona pelúcida ${ }^{15}$. $\mathrm{O}$ aumento da concentração de células inflamatórias na cavidade peritoneal causa dano não somente aos oócitos e aos espermatozoides, mas também tem sido mostrados efeitos tóxicos ao embrião em formação ${ }^{13}$. Nessa perspectiva, estudos relatam expressão aberrante da enzima glutationa peroxidase e catalase no endométrio tópico de pacientes com endometriose associada ao aumento local de radicais livres, levando a efeitos negativos diretos na viabilidade do embrião ${ }^{13,18}$.

\section{Discussão}

A endometriose é reconhecida como uma das patologias mais comuns dentro da ginecologia moderna ${ }^{12}$ e permanece uma doença controversa não somente pela complexidade de sua fisiopatologia e dificuldade na compreensão de sua evolução natural, mas também por sua relevância como fator predisponente à infertilidade ${ }^{19}$. Segundo Miller et al. $^{6}$, existem 
diversos mecanismos interligados envolvidos no surgimento dos quadros de infertilidade associada à doença, sendo a inflamação peritoneal componente central de todos os eventos patológicos.

Acredita-se que em graus mais avançados da doença as alterações da anatomia pélvica são significativas no sentindo de explicar a relação do quadro com a infertilidade, principalmente por meio das adesões, que podem prejudicar, entre outros aspectos, a liberação do oócito na cavidade peritoneal bem como sua captação pelas tubas uterinas. Contudo, nos casos de endometriose leve e moderada associados à infertilidade, ainda permanecem grandes lacunas no que diz respeito aos mecanismos fisiopatológicos envolvidos, sendo descritos na literatura fatores como foliculogênese prejudicada e defeitos de implantação relacionados principalmente a fatores inflamatórios situados nos fluidos folicular e peritoneal ${ }^{19}$.

O processo inflamatório presente na cavidade peritoneal de pacientes com endometriose ocasiona aumento significativo nos níveis locais de citocinas, prostaglandinas e, consequentemente, promove um estado de constante estresse oxidativo celular que pode prejudicar a interação entre oócito e espermatozoide, dificultando o desenvolvimento do embrião e o processo de implantação no endométrio tópico ${ }^{14}$. Nessa perspectiva, nota-se também um aumento intrafolicular nos níveis de interleucina 8, interleucina 12 e adrenomedulina, peptídeo presente em quadros inflamatórios como sepse, em mulheres submetidas à fertilização in vitro já diagnosticadas com endometriose, o que indica qualidade prejudicada dos oócitos e do futuro embrião ${ }^{6}$. Ademais, estudos da literatura mais recente sugerem que componentes presentes no líquido peritoneal de pacientes com endometriose possuam a capacidade de levar à imobilização dos espermatozoides, principalmente através da ação de macrófagos ${ }^{11,18}$. Interleucinas 1 e 6 também atuam diretamente na mobilidade dos gametas e TNF-alfa causa dano ao DNA dos espermatozoides de maneira concentraçãodependente e tempo-dependente, possivelmente por meio da ação de espécies reativas de oxigênio e apoptose exacerbada ${ }^{15}$. Além disso, há alteração na peristalse útero-tubária que pode contribuir para a infertilidade feminina uma vez que ocasiona distúrbios no transporte embrionário através de movimentos descoordenados ${ }^{14}$. Estudo de Lyons et al. ${ }^{20}$ 
mostrou que a frequência de batimentos de células ciliares nas tubas uterinas de mulheres com endometriose se mostra menor em relação a pacientes saudáveis, comparando amostras de tubas uterinas oriundas de histerectomias e incubadas em fluido peritoneal de mulheres com e sem doença ${ }^{20}$.

De acordo com Tanbo e Fedorcsak ${ }^{14}$, mulheres com endometriose apresentam maior prevalência de síndrome LUF (luteinized unruptured follicle), condição em que o folículo dominante falha no processo de ruptura e não promove a liberação do oócito, mesmo havendo aumento da temperatura corporal basal e aumento da progesterona nessas pacientes ${ }^{21}$. Ademais, anti-inflamatórios não esteroidais, que frequentemente são utilizados como tratamento de primeira linha para dismenorreia secundária associada à endometriose, também estão associados a um maior risco de desenvolvimento da síndrome ${ }^{14}$.

Estudo feito por Miller et al. ${ }^{6}$, encontrou 91 genes de expressão aberrante no endométrio tópico de pacientes inférteis diagnosticadas com endometriose. Os genes expressos eram predominantemente envolvidos nos processos de adesão celular, interação entre citocinas, apoptose e decidualização. Aumento de células natural killers uterinas, subtipos de células natural killer CD16+ envolvidas na patogênese de importantes doenças obstétricas como préeclâmpsia e restrição do crescimento intrauterino, são encontradas no endométrio de pacientes com a doença. ${ }^{22}$

Alterações hormonais observadas em mulheres com endometriose podem ser divididas em dois tipos: produção local anormal de estrogênio relacionada à inflamação e resistência aos efeitos da progesterona ${ }^{15}$. O desequilíbrio hormonal verificado em pacientes com diagnóstico de endometriose ocorre devido à expressão aumentada da enzima aromatase (CYP19A1) e expressão diminuída de 17ßhidroxisteroide desidrogenase regulada pela progesterona, dentre outros mecanismos, o que resulta em níveis aumentados de estradiol (E2) biodisponível nos ovários, tecidos periféricos e nas lesões endometrióticas e resistência à progesterona ${ }^{23}$. Tais alterações provocam danos inflamatórios diretos ao endométrio tópico e resultam em ciclos anovulatórios, uma vez que a secreção adequada de progesterona, assim como a responsividade do endométrio tópico a sua ação durante a fase lútea do ciclo menstrual são mandatórias para a transição de um endométrio 
proliferativo para um endométrio em estado secretório e receptivo ${ }^{14}$.

Além disso, há dados na literatura recente sugerindo que pacientes diagnosticadas com endometriose apresentam concomitantemente quadro de hiperprolactinemia ${ }^{26,27}$, condição que por inibir a secreção apropriada de hormônio liberador de gonadotrofinas $(\mathrm{GnRH}), \quad$ resulta $\mathrm{em}$ hipogonadismo e anovulação ${ }^{1 .}$ Nesse sentido, existem estudos promissores que buscam relacionar a dosagem da prolactina com a função de possível biomarcador da doença que, associado ao CA-125, poderia ser útil para seguimento das pacientes e diagnóstico precoce de recidivas da doença ${ }^{27}$.

Ademais, evidências sugerem que há diminuição da expressão endometrial da integrina $\alpha \mathrm{v}-\beta$, molécula que atua diretamente no processo de adesão celular, durante o período de implantação em mulheres com endometriose ${ }^{24}$. A falha na implantação do embrião também pode ser resultado de níveis elevados de óxido nítrico endotelial, que pode causar efeitos citotóxicos que influenciam de maneira negativa na receptividade do endométrio tópico ao embrião, e L-selectina, proteína que impede o blastocisto de encontrar o local apropriado para adesão ao endométrio ${ }^{25}$.

A endometriose é considerada uma das principais causas do desenvolvimento de adesões não relacionadas a procedimentos

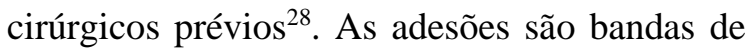
tecido conjuntivo que ligam duas estruturas diferentes que normalmente são separadas, interferindo na função dos órgãos afetados. Com isso, essa condição pode prejudicar a fertilidade espontânea por meio de barreiras mecânicas à ovulação e ao desenvolvimento embrionário na cavidade uterina, principalmente nos casos mais graves da doença $a^{19,28,29,30}$.

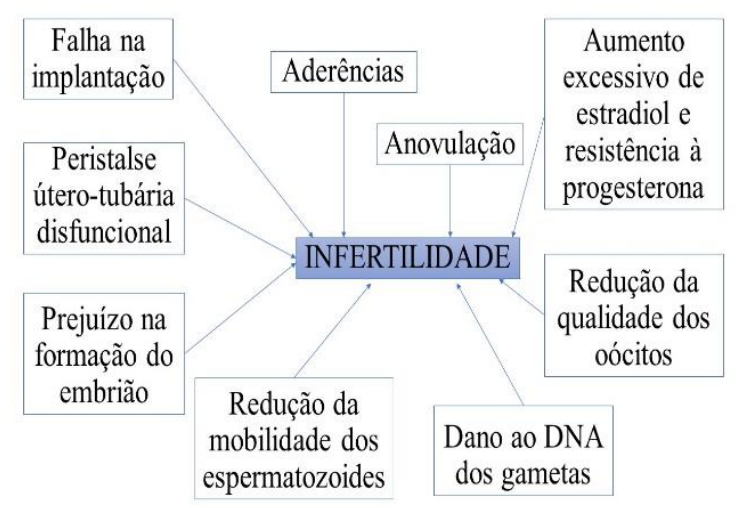

Figura 1 - Mecanismos envolvidos na patogênese da infertilidade por endometriose

\section{Conclusão}

Com base nas informações obtidas por meio dos estudos analisados acerca do tema, observou-se que o ambiente inflamatório gerado pelos focos de endométrio ectópico atua como 
componente central de todos os processos fisiopatológicos que envolvem a complexa relação entre a endometriose e a infertilidade feminina. As respostas inflamatórias locais levam a importantes prejuízos nas funções essenciais à concepção espontânea como ovulação, por meio de desequilíbrio na secreção de hormônios como estrogênio, progesterona, gonadotrofinas e prolactina, interação adequada entre oócito e espermatozoide, transporte coordenado do embrião e implantação do embrião na cavidade uterina.

Apesar dos atuais questionamentos levantados a respeito do mecanismo etiológico da infertilidade relacionada à endometriose ainda não apresentarem respostas exatas, observou-se grande quantidade de estudos disponíveis na literatura acerca do tema apresentado, com destaque àqueles que buscam identificar os principais componentes da resposta inflamatória envolvida. Dessa forma, esperam-se novos resultados promissores a fim de elaborar protocolos de tratamento com alvos específicos para aumentar as chances de uma possível gestação espontânea e melhorar a qualidade de vida das pacientes que possuam o desejo de gestar.

\section{Referências}

1. Freitas F. Rotinas em Ginecologia. 6. ed. São Paulo: Artmed; 2011. 730p.

2. Burney RO, Giudice LC. Pathogenesis and pathophysiology of endometriosis. Fertility and Sterility. 2012 September; 98(3): 51119.

3. Borghese B, Zondervan KT, Abrao MS, Chapron C, Vaiman D. Recent insights on the genetics and epigenetics of endometriosis. Clinical Genetics. 2017; 91: 254-64.

4. Jorgensen H, Hill AS, Beste MT, Kumar MP, Chiswick E, Fedorcsak $\mathrm{P}$, et al. Peritoneal fluid cytokines related to endometriosis in patients evaluated for infertility. Fertility and Sterility. 2017 May; 107 (5): 1191-99.

5. Podgaec S. Manual de Endometriose. 1. ed. São Paulo: Federação Brasileira das Associações de Ginecologia e Obstetrícia (FEBRASGO); 2014. 104p.

6. Miller JE, Ahn SH, Monsanto SP, Khalaj K, Koti M, Tayade C. Implications of imune dysfunction on endometriosis associated infertility. Oncotarget. 2017 October; 8 (4): 7138-47.

7. Viganò $\mathrm{P}$, Parazzini F, Somigliana E, Vercellini P. Endometriosis: epidemiology and aetiological factors. Best Practice \& Research Clinical Obstetrics and Gynaecology. 2004; 18 (2): 177-200.

8. Wei W, Zhang X, Tang H, Zeng L, Wu R. Microbiota composition and distribution along the female reproductive tract of women with endometriosis. Annals of Clinical Microbiology and Antimicrobials. 2020; 1-8.

9. Tomás C, Metello JL. Endometriose e infertilidade - onde estamos? Acta Obstétrica e Ginecológica Portuguesa. 2019; 13 (4): 235-41. 
10. Canis M, Donnez JG, Guzick DS, Halme JK, Schenken RS, Rock JÁ, et al. Revised American Society for Reproductive Medicine classification of endometriosis: 1996. Fertility and Sterility. 1997; 67 (5): 817-21.

11. Navarro PA. Infertility Associated to Endometriosis: Clarifying Some Important Controversies. Revista Brasileira de Ginecologia e Obstetrícia. 2019; 41: 523-24.

12. Vila ACD. A endometriose e sua relação com a infertilidade feminina e fatores ambientais [master's thesis]. Goiânia: Universidade Católica de Goiás; 2007. 71p.

13. Macer ML, Taylor HS. Endometriosis and Infertility - A Reviw of the Pathogenesis and Treatment of Endometriosis-associated Infertility. Obstetrics and Gynecology Clinics of North America Special Issues. 2012; 39: 535-49.

14. Tanbo T, Fedorcsak P. Endometriosisassociated infertility: aspacts of pathophysiological mechanisms and treatment options. Acta Obstetricia et Gynecologica Scandinavica. 2017; 96: 65967.

15. Ziegler D, Borhese B, Chapron C. Endometriosis and infertility: pathophysiology and management. The Lancet. 2010; 376: 730-38.

16. Poppe ACM. Regulação da Resposta Imune e Endometriose [undergraduate thesis]. São Paulo: Universidade Estadual Paulista; 2008. 20p.

17. Santos BP. Endometriose e sua influência na fertilidade feminina [undergraduate thesis]. Goiânia: Universidade Federal de Goiás; 2016. 36p.

18. The Practice Committee of the American Society for Reproductive Medicine. Fertility and Sterility. 2006 November; 86: 156-60.

19. Marques, MR. Endometriose e infertilidade: revisão sistemática da literatura e relato de casos [undergraduate thesis]. Florianópolis:
Universidade Federal de Santa Catarina; 2005. 111p.

20. Lyons RA, Djahanbakhch O, Saridogan E, Naftalin AA, Mahmood T, Weekes A, et al. Peritoneal fluid, endometriosis, and cilliary beat frequency in the human fallopian tube. The Lancet. 2002 October; 360: 1221-22.

21. Passos EP, Freitas F, Filho JSC, Facin AC, Souza CAB, Salazar CC. Endometriose. Revista HCPA. 2000; 20 (2): 150-56.

22. Ahn SH, Khalaj K, Young SL, Lessey BA, Koti M, Yayade C. Immune-inflammation gene signatures in endometriosis patients. Fertility and Sterility. 2016: 11-19.

23. Gómez EG, Martinez ERV, Mayoral CR, Orozco OPC, Arroyo IG, Cerbón M. Regulation of Inflammation Pathways and Inflammasome by Sex Steroid Hormones in Endometriosis. Frontiers in Endocrinology. 2020 January; 10: 1-17.

24. Lessey BA, Castelbaum AJ, Sawin SW, Buck CA, Schinnar R, Bilker W, et al. Aberrant Integrin Expression in the Endometrium of Women with Endometriosis. Journal of Clinical Endocrinology and Metabolism. 1994; 79 (2): 643-49.

25. Santos DB, Soares IA, Filho LAF, Fernandes MDB, Ritt NMM, Barcelos RP, et al. Uma abordagem integrada da endometriose. 1. ed. Cruz das Almas/BA: Editora UFRB; 2012. 120p.

26. Mirabi P, Alamolhoda SH, Golsorlhtabaramiri M, Namdari M, Esmaeilizadeh S. Prolactin concentration in various stages of endometriosis in infertile women. JBRA Assisted Reproduction. 2019; 23 (3): 225-29.

27. De Conto E, Cunha-Filho JSL, Alfonsin M, Bilibio JP, Souza CAB. Avaliação dos níveis séricos de prolactina e CA-125 como biomarcadores para o diagnóstico da endometriose. In: Semana Científica do HCPA. Porto Alegre: Universidade Federal do Rio Grande do Sul. 
28. El-kader AIA, Gonied AS, Mohamed ML, Mohamed SL. Impact of Endometriosisrelated Adhesions on Quality of Life among Infertile Women. International Journal of Fertility and Sterility. 2019; 13 (1): 72-76.

29. May KE, Conduit-Hulbert SA, Villar J, Kirtley S, Kennedy SH, Becker CM. Peripheral biomarkers of endometriosis: a systematic review. Human Reproduction Update. 2010; 16 (6): 651-74.
30. Evans MB, Decherney AH. Fertility and Endometriosis. Clinical Obstetrics and Gynecology. 2017; 60 (3): 497-502.

\section{Reservado aos Editores}

Data de submissão: 04/02/2021

Data de aprovação: 28/04/2021 